\title{
Conocimientos y prácticas sobre métodos de planificación familiar en adolescentes escolarizados de Cartagena-Bolívar.
}

\author{
Knowledge and practices of family planning methods of school adolescents from \\ Cartagena-Bolivar
}

\section{Conhecimentos e práticas sobre métodos de planejamento familiar em adolescentes educados na cidade de Cartagena, Bolívar}

\author{
Moraima Del Toro-Rubio* \\ Keydis Sulay Ruidiaz-Gómez ${ }^{* *}$ \\ Zorayda Barrios-Puerta***
}

\begin{abstract}
Autor de correspondencia
* $\bowtie$ Enfermera. Magister en educación. Docente de la Corporación Universitaria Rafael Núñez. Universidad de Cartagena. Correo: moraima.deltoro@curnvirtual.edu. co. (iD) https://orcid.org/0000-0001-67105171. Cartagena, Colombia.

** Enfermera. Magister en enfermería. Docente de la escuela de enfermería. Universidad del Sinú Elías Bechara Zainúm Cartagena. Estudiante de Doctorado en Enfermería. Correo: coordinvestenfermeria@unisinucartagena.edu.co. (iD https://orcid.org/00000001-9335-8930. Cartagena, Colombia.

*** Enfermera. Magister en educación. Docente de la Corporación Universitaria Rafael Núñez, Universidad del Sinú Elías Bechara Zainúm Cartagena. Correo: zorayda.barrios@curnvirtual.edu.co. (ID) https://orcid. org/0000-0001-8532-4511. Cartagena, Colombia.
\end{abstract}

Este es un artículo bajo la licencia CC BY

(https://creativecommons.org/

licenses/by/4.0/) @) (i)

\section{Resumen}

Introducción: En Colombia se capacitan a parejas, madres cabeza de familia y jóvenes adolescentes sobre los beneficios de la planificación familiar, con el objeto de lograr una vida sexual segura y satisfactoria, de tal forma que se tenga conciencia con el fin de decidir en qué momento y frecuencia se logra la procreación. Objetivo: Identificar el conocimiento y la práctica que tienen sobre métodos de planificación familiar los estudiantes de una Institución Educativa. Materiales y Métodos: Estudio descriptivo, transversal y correlacional. La muestra estuvo conformada por 229 estudiantes (noveno: 38,9\%, décimo: 33,6\% y undécimo: 27,4\%), seleccionada de manera aleatoria simple; se utilizó la encuesta de medición de conocimientos y prácticas sobre métodos de planificación familiar. Resultados: La Edad osciló entre 16 y 18 años y corresponde al $51,1 \%$ de estudiantes. Están distribuidos como sigue: hombres $47,6 \%$, mujeres $52 \%$, solteros $82,1 \%$, urbanos $82,1 \%$, viven con la madre $81,7 \%$ y el $61,8 \%$ habla de planificación familiar con la madre. Conocen sobre métodos anticonceptivos $(94,8 \%)$, han recibido información de su uso $(92,1 \%)$, en el colegio $(39,3 \%)$ y en la familia $(14,4 \%)$. Por otra parte, indicaron que el método que consideran previene el embarazo e ITS es el condón ( $86 \%$ ), y que en la primera relación sexual puede sobrevenir el embarazo $(71,2 \%)$. Del mismo modo, por pena o vergüenza no solicitan información sobre los métodos anticonceptivos (51,5\%), algunos no han tenido su primera relación sexual (50,7\%); también manifestaron que el primer método de planificación familiar empleado fue el condón (67\%). La mayor información sobre: conocimientos, prácticas y aspectos sociodemográicos se relacionó entre sí $(p<0,05)$. Conclusiones: El conocimiento, las prácticas y las diversas características sociodemográficas repercuten en muchos aspectos de la salud sexual y reproductiva de estos estudiantes.

Palabras Claves: Planificación Familiar, Salud Sexual y Reproductiva, Adolescente, Estudiantes. 


\begin{abstract}
Introduction: In Colombia, couples, matriarchs and young adolescents are educated about the benefits of family planning, with the objective of achieving a safe and satisfactory sexual life. The purpose is to have awareness in order to decide when and how frequently they wish to reproduce. Objective: Identify the knowledge and practice students from an Educational Institution have about the contraception methods. Materials and Methods: The sample was composed of 229 students (ninth grade: $38.9 \%$, tenth grade: $33.6 \%$, and eleventh grade: $27.4 \%$ ) randomly selected; the survey of measurement of knowledge and practices about family planning methods was used. Results: The age range was between 16 and 18 years old and corresponds to $51.1 \%$ of the students. These were distributed as follows: $47.6 \%$ men, $52 \%$ women, $82.1 \%$ single, $82.1 \%$ urban, $81.7 \%$ live with their mother and $61.8 \%$ talk about family planning with their mother. $94.8 \%$ know about contraception methods, $92.1 \%$ have received information about their use (39.3\% at school and 14.4\% at home). Additionally, $86 \%$ of the students indicated that condoms prevent pregnancy and STI's, and $71.2 \%$ indicate that pregnancy can occur on the first sexual encounter. Also, because of shame or embarrassment, $51.5 \%$ of students do not ask for information about contraception methods; $67 \%$ also manifested that the first contraception method used by them was the condom. Most of the information about knowledge, practices and socio-demographic aspects were related to each other $(p<0.05)$. Conclusions: The knowledge, practices and diverse socio-demographic characteristics have an impact in many aspects of sexual and reproductive health in these students.
\end{abstract}

Keywords: Family Planning, Sexual and Reproductive Health, Adolescent, Students.

\title{
Resumo
}

Introdução: Na Colômbia se capacitam os casais, mulheres chefes de família e jovens adolescentes sobre os benefícios do planejamento familiar, com o objeto de obter uma vida sexual segura e satisfatória, de tal maneira que se tenha consciência com o fim de decidir em que momento e frequência se consegue a procriação. Objetivo: Identificar o conhecimento e a prática que tem sobre métodos de planejamento familiar os alunos de uma Instituição Educativa na cidade de Cartagena, Bolívar. Materiais e Métodos: Estudo descritivo, transversal e correlacional. A amostra esteve conformada por 229 alunos (nona série do ensino fundamental: 38,9\%, primeira seria de ensino médio: 33,6\% e segunda série do ensino médio: 27,4\%), selecionada de maneira aleatória simples; utilizou-se a pesquisa de medição de conhecimentos e práticas sobre métodos de planejamento familiar. Resultados: A idade oscilou entre 16 e 18 anos e correspondeu ao 51,1\% dos alunos. Estiveram distribuídos como segue: homes $47,6 \%$, mulheres $52,4 \%$, solteiros $82,1 \%$, urbanos $82,1 \%$, que moram com a mãe $81,7 \%$ e que falam de planejamento familiar com a mãe $61,8 \%$. Conhecem sobre métodos anticoncepcionais $(94,8 \%)$, tem recebido informação de seu uso $(92,1 \%)$, na escola $(39,3 \%)$ e na família $(14,4 \%)$. Por outra parte, indicaram que o método que consideram previne a gravidez e as Infecções Sexualmente Transmissíveis (IST) é a camisinha (86\%), e que no primeiro relacionamento sexual a gravidez pode ocorrer $(71,2 \%)$. Do mesmo modo, por vergonha ou constrangimento não solicitam informação sobre os métodos anticoncepcionais (51,5\%), alguns não tem tido seu primeiro relacionamento sexual (50,7\%); também manifestaram que o primeiro método de planejamento familiar empregado foi a camisinha (67\%). A maior informação sobre: conhecimentos, práticas e aspectos sociodemográficos se relacionou entre si $(p<0,05)$. Conclusões: O conhecimento, as práticas e as diversas características sociodemográficas repercutem em muitos aspectos da saúde sexual e reprodutiva destes alunos.

Palavras-chave: Adolescente, Estudantes, Planejamento Familiar, Saúde Sexual e Reprodutiva

\section{Introducción}

El concepto de planificación familiar, en esencia, esta direccionado al control de la natalidad en una comunidad, región o país. Por ello, se hace necesario que la pareja esté preparada económica, social y sicológicamente para procrear; es decir, que debe disponer de los suficientes recursos para tener una vida digna y así gozar de las oportunidades que le ofrece el entorno hoy día (1). 
La Organización Mundial de la Salud (OMS) define la Planificación Familiar (PF) como:

"una manera de pensar y de vivir que es adoptada voluntariamente sobre la base de conocimientos, actitudes y decisiones responsables por parte de individuos y parejas, con el fin de promover la salud y el bienestar del grupo familiar, y por lo tanto, contribuir de manera eficaz al desarrollo social de un país" (2).

Por otra parte, la Encuesta Nacional de Demografía y Salud (ENDS) de 2015 confirma que el porcentaje de adolescentes de 15 a 19 años, madres o embarazadas del primer hijo o hija disminuyó pasando del 19,5 \% en 2010 al 17,4\% en 2015, es decir, que hubo un descenso del porcentaje de madres embarazadas del primer hijo en todos sus niveles de desagregación: edad, zona, región, nivel educativo y quintil de riqueza, siendo mayor la tendencia entre las mujeres de 19 años (3).

En Colombia, la tasa de fecundidad ha disminuido sistemáticamente de 6,7 hijos en 1967 por mujer a 2 en el 2015 según la tasa global de fecundidad(3). Desde esta perspectiva, el país ha sido considerado como una de las comunidades latinoamericanas más exitosa en lograr un descenso rápido de la fecundidad; sin embargo, a pesar de la alta prevalencia en el uso de métodos, existe una gran demanda insatisfecha, especialmente en las poblaciones rurales y en las urbanas menos favorecidas. Teniendo en cuenta lo planteado anteriormente la ENDS 2015 reporta que: el $80,9 \%$ de las mujeres y el $82,7 \%$ de los hombres entre 13 y 49 años usan algún método anticonceptivo; mientras, que el $34,9 \%$ de las mujeres se ha realizado la esterilización: el $36,2 \%$ en zona urbana y el 31,1\% en la rural (3).

Sin embargo, se debe tener en cuenta que la conducta sexual y reproductiva de un adolescente está influenciada por su contexto sociocultural, así como por las percepciones sobre su bienestar psicoafectivo, lo que lleva a un planteamiento relacionado con las necesidades que suplen y crean un concepto de sexualidad $(4,5)$. Para Lapeira et al. (6), uno de los factores que determina la fecundidad en una población es la edad en que la mujer inicia su vida reproductiva, factor que afecta a su vez los índices de mortalidad materna y mortalidad infantil, lo que supone que entre más temprano el adolescente inicie su vida sexual más inadecuado será el manejo de su sexualidad, debido fundamentalmente a los limites en la comunicación, las dificultades frente a las relaciones sexuales y el uso de métodos anticonceptivos (6).

Actualmente, en Colombia las políticas en salud conjuntamente con el Ministerio de Educación trabajan para ofrecer educación sexual en las instituciones educativas, como una herramienta para disminuir los índices de fecundidad y fomentar una sexualidad responsable (7).

Se debe precisar, que la planificación familiar vista desde un horizonte integral contribuye a mejorar la calidad de vida de mujeres, niños y hombres de una comunidad, siempre que el país efectúe las inversiones que posibiliten el bienestar y el mantenimiento de la salud (8). Aunque la información sobre planificación familiar es amplia, aún existen brechas en la población adolescente relacionadas con: falta de información, uso inadecuado de métodos, abortos y embarazos no planificados, factores que limitan el futuro de un adolescente y agrava las situaciones económicas de un país (9).

Por otra parte, el conocimiento y la práctica de los adolescentes sobre la anticoncepción merece atención especial en todos los niveles escolares, donde generalmente los embarazos no son planificados y terminan en abortos practicados en condiciones sanitarias inapropiadas e incluso el abandono por parte de la familia tanto de la mujer como la del hijo, lo que incrementa los problemas sociales y sanitarios de la población $(10,11)$.

En Cartagena, el Departamento Administrativo Distrital de Salud (DADIS), aborda estrategias para mejorar la salud sexual y reproductiva, al mismo tiempo, promueve el ejercicio de los derechos sexuales y reproductivos; por consiguiente, con esta investigación se aspira a contribuir a la disciplina de enfermería, aportando herramientas conceptuales que permitan realizar un abordaje integral sobre la situación de los adolescentes frente a la práctica responsable de su sexualidad; de igual manera, se pretende que mediante la inclusión de esta temática en el currículo de enfermería proporcione elementos de intervención, planificación y abordaje específico en una región.

Del mismo modo, la contribución de otras disciplinas aportarán aspectos relacionados con la atención holística y longitudinal, permitiendo el liderazgo en la gestión social en los programas de salud relacionados con el cuidado y prevención primaria de este grupo poblacional. En este orden de ideas, es 
importante resaltar que el trabajo interdisciplinario genera propuestas de intervención y de integración de acciones efectivas, necesarias para el logro de los objetivos en materia de salud sexual y reproductiva. Así mismo, los hallazgos demuestran que si existe una autoevaluación de las prácticas en adolescentes frente al uso de métodos anticonceptivos y sus conductas de autocuidado, favorecen el desarrollo de conocimientos y prácticas sanitarias que contribuyan al fomento de estilos de vida saludables.

\section{Objetivos}

\section{Objetivo General}

Determinar los conocimientos y prácticas sobre métodos de planificación familiar en estudiantes de una Institución Educativa.

\section{Objetivos Específicos}

- Caracterizar la población según: edad, sexo, procedencia social, nivel educativo, tipo de familia y número de hermanos.

- Identificar las variables sobre conocimiento y práctica de los estudiantes frente a los métodos de planificación familiar.

- Establecer asociaciones entre las variables de conocimientos y prácticas con las características demográficas.

\section{Materiales y Métodos}

Para esta investigación se adoptó un enfoque descriptivo transversal correlacional $(12,13)$.

La población estuvo conformada por 570 estudiantes de una Institución Educativa de la ciudad de Cartagena
Y la muestra por 229, distribuidos en los grados noveno, décimo y undécimo. Se tomaron en cuenta los siguientes criterios de inclusión: ser estudiante de los grados académicos seleccionados, contar con el aval de los padres y participar por voluntad propia. La fórmula de cálculo contó con una Desviación estándar: 1.6, nivel de confianza: 95\%, margen error de muestra: $5 \%$ y el muestreo fue aleatorio simple.

La recolección de la información se realizó durante los meses de febrero y noviembre de 2016, la misma consistió en la aplicación de un instrumento creado por los investigadores, titulado "conocimientos y prácticas sobre métodos de planificación familiar"; esta encuesta en cuanto a su contenido fue validada por el juicio de expertos; asimismo la prueba piloto obtuvo un índice de confiabilidad Alpha Cronbach igual a $0.65 \mathrm{~s}$. El instrumento consta de 30 preguntas dicotómicas, distribuidas así: 7 ítems para aspectos sociodemográficos, 11 interrogantes para conocimientos y 12 preguntas para el componente de prácticas.

Los datos fueron almacenados en una hoja de cálculo Microsoft Excel para su posterior análisis a través del programa estadístico SPSS 20, con licencia para el grupo de investigación. Los resultados se representaron en tablas y cálculos de frecuencias absolutas y relativas.

La existencia de asociación se determinó por Chi Cuadrado (Chi2), en caso de existir asociación, posteriormente se procedió a calcular su magnitud, empleando la herramienta estadística conocida como V de Cramer (ver Tabla 1). Una vez estimado el anterior coeficiente se procedió a calcular la probabilidad.

Tabla1. Intensidad de la correlación

\begin{tabular}{cc}
\hline VALORES DE V DE CRAMER & $\begin{array}{c}\text { INTERPRETACIÓN DE LA } \\
\text { CORRELACIÓN }\end{array}$ \\
\hline Entre 0 y 0,25 & Escasa \\
Entre 0,25 y 0,5 & Cierto grado de correlación \\
Entre 0,5 y 0,75 & Relación de moderada a buena \\
Mayores a 0,75 & Relación de buena a excelente \\
\hline
\end{tabular}

Fuente: Rodríguez et al. (14). 
ISSN-PRINT

1794-9831

E-ISSN 2322-7028

Vol. 15 No. 2

Jul - Dic 2018

Cúcuta, Colombia
Desde el punto de vista ético debe señalarse que se tuvo en cuenta la Resolución 008430 de 1993 (15), la cual sirvió de guía ética para la realización del presente estudio, considerado sin riesgo debido a que los datos se obtuvieron sin llevar a cabo experimentos sobre los sujetos. Igualmente, se contó con el consentimiento de los padres de familia y un formato de aprobación para que sus hijos participaran como informantes de este estudio por ser menores de edad; material que contenía información clara y completa del estudio, de tal forma que el padre o el representante legal la comprendiera y la firmara de forma voluntaria.

El estudio se realizó con fines académicos, con total reserva y respeto al participante y sus familiares; asimismo, fue sometido a la aprobación del comité de ética de la institución bajo el código de proyecto CURN 04-2016.

\section{Resultados}

\section{Características sociodemográficas}

El 38,9 \% de los estudiantes pertenecen a noveno grado, $33,6 \%$ a décimo y $27,5 \%$ a undécimo, con edades comprendidas entre 16-18 años (51,1\%); siendo el $52 \%$ mujeres solteras procedentes de la zona urbana. Con respecto a la tipología familiar se observó un predominio de familias monoparentales en los grados noveno (83,1\%), décimo $(89,6 \%)$ y undécimo $(69,8 \%)$, siendo la madre el miembro principal (Ver tabla 2)

Tabla 2. Aspectos sociodemográficos de los estudiantes.

\begin{tabular}{|c|c|c|c|c|c|c|c|c|c|}
\hline \multirow[t]{2}{*}{ Variable } & \multirow[t]{2}{*}{ Ítem } & \multicolumn{4}{|c|}{ Frecuencia } & \multicolumn{4}{|c|}{ Porcentaje } \\
\hline & & Noveno & Décimo & Undécimo & Total & Noveno & Décimo & Undécimo & Total \\
\hline \multirow{4}{*}{ Edad } & $13-15$ & 58 & 29 & 14 & 101 & $65,20 \%$ & $37,70 \%$ & $22,20 \%$ & $44,10 \%$ \\
\hline & $16-18$ & 28 & 47 & 42 & 117 & $31,50 \%$ & $61,00 \%$ & $66,70 \%$ & $51,10 \%$ \\
\hline & $19-21$ & 1 & 1 & 7 & 9 & $1,10 \%$ & $1,30 \%$ & $11,10 \%$ & $3,90 \%$ \\
\hline & No responde & 2 & 0 & 0 & 2 & $2,20 \%$ & $0,00 \%$ & $0,00 \%$ & $0,90 \%$ \\
\hline \multirow{3}{*}{ Sexo } & Femenino & 45 & 40 & 34 & 119 & $50,60 \%$ & $51,90 \%$ & $54,00 \%$ & $52,00 \%$ \\
\hline & Masculino & 44 & 37 & 28 & 109 & $49,40 \%$ & $48,10 \%$ & $44,40 \%$ & $47,60 \%$ \\
\hline & No responde & 0 & 0 & 1 & 1 & $0,00 \%$ & $0,00 \%$ & $1,60 \%$ & $0,40 \%$ \\
\hline \multirow{4}{*}{ Estado civil } & Soltero & 70 & 67 & 51 & 188 & $78,70 \%$ & $87,00 \%$ & $81,00 \%$ & $82,10 \%$ \\
\hline & Casado & 2 & 1 & 1 & 4 & $2,20 \%$ & $1,30 \%$ & $1,60 \%$ & $1,70 \%$ \\
\hline & Unión libre & 10 & 8 & 8 & 26 & $11,20 \%$ & $10,40 \%$ & $12,70 \%$ & $11,40 \%$ \\
\hline & No responde & 7 & 1 & 3 & 11 & $7,90 \%$ & $1,30 \%$ & $4,80 \%$ & $4,80 \%$ \\
\hline \multirow{3}{*}{ Procedencia } & Rural & 7 & 2 & 3 & 12 & $7,90 \%$ & $2,60 \%$ & $4,80 \%$ & $5,20 \%$ \\
\hline & Urbana & 67 & 65 & 56 & 188 & $75,30 \%$ & $84,40 \%$ & $88,90 \%$ & $82,10 \%$ \\
\hline & No responde & 15 & 10 & 4 & 29 & $16,90 \%$ & $13,00 \%$ & $6,30 \%$ & $12,70 \%$ \\
\hline \multirow{4}{*}{ Tipología familiar } & Monoparental & 74 & 69 & 44 & 187 & $83,10 \%$ & $89,60 \%$ & $69,89 \%$ & $81,70 \%$ \\
\hline & Extensa & 56 & 40 & 32 & 128 & $62,90 \%$ & $51,90 \%$ & $50,80 \%$ & $55,90 \%$ \\
\hline & Nuclear & 50 & 42 & 27 & 119 & $56,20 \%$ & $54,50 \%$ & $42,90 \%$ & $52,00 \%$ \\
\hline & Total & 89 & 77 & 63 & 229 & $100,00 \%$ & $100,00 \%$ & $100,00 \%$ & $100,00 \%$ \\
\hline
\end{tabular}

Fuente: Instrumento acerca de los conocimientos y las prácticas sobre métodos de planificación familiar en una institución educativa

\section{Conocimientos de los estudiantes sobre métodos de Planificación Familiar.}

El 94,8 \% de los estudiantes de la institución dicen conocer los métodos anticonceptivos, distribuidos así: grado noveno $93,3 \%$, décimo $93,5 \%$ y undécimo $98,4 \%$. El $92,1 \%$ ha recibido información sobre el tema, distribuidos como sigue: $94,4 \%$ de noveno, $93,5 \%$ de décimo y el $87,3 \%$ de undécimo. Con respecto a la información recibida se comprobó que los estudiantes de undécimo la valoran como muy positiva (54\%), los de décimo con un promedio medio (41,6 $\%$; además, se evalúa con calificaciones excelente y regular $(22,1 \%)$. En contraste, entre los estudiantes de noveno se observaron las mejores valoraciones: $36 \%$ y $46,1 \%$ respectivamente. Las fuentes de información primaria sobre métodos de planificación familiar son 
las siguientes: el colegio con un 43,8 \%, entre los estudiantes de undécimo y noveno grado el 52,4 \%, y el $93 \%$ exponen haber recibido información sobre métodos anticonceptivos alguna vez en su vida. Por otro lado, el $86 \%$ de los estudiantes coincidieron en afirmar que el método anticonceptivo que previene de un embarazo y enfermedades de trasmisión sexual es el condón; el rango de edad del uso del anticonceptivo se encuentra entre 16 a 20 años $(65,5 \%)$; de igual manera reconocieron que en la primera relación sexual se puede quedar embarazada (71,2\%). Asimismo, un $74,7 \%$ reconoce que los embarazos a temprana edad se consideran de alto riesgo; con respecto al concepto de planificación familiar, un 48,9\% de adolescentes refieren que planificar es "controlar la reproducción mediante el uso de anticonceptivos". Cabe destacar, que los métodos anticonceptivos más conocidos por los estudiantes son: el condón con una valoración alta entre los de noveno y décimo: 98,8 \% y 98,7 \%; seguido de la píldora, noveno: $80 \%$, décimo: 69,7 \% y undécimo: $88,7 \%$.

\section{Prácticas de los estudiantes con relación a los métodos de Planificación Familiar}

Respecto a la práctica, los encuestados manifestaron que no solicitan información sobre métodos anticonceptivos por pena o vergüenza $(51,5 \%)$; el $62,9 \%$ pertenece al grado noveno, el $39 \%$ al décimo y el 50,8\% al undécimo; sin embargo, el $83,9 \%$ reconoció que la planificación familiar es un asunto importante.

Con relación al ítem: ha empezado a tener relaciones sexuales, respondieron afirmativamente en todos los grados (noveno 37,1 \% y décimo 37,7 \%); cabe resaltar, que el $54 \%$ de estudiantes de grado undécimo manifestó haber iniciado su vida sexual a una edad muy temprana. Es de hacer notar, que el 65,6 $\%$ manifestó que el primer método de planificación utilizado por él y su pareja fue el condón, igualmente, expresaron que el método que generalmente utilizan es el condón con $70,8 \%$.

Cabe destacar, que los estudiantes expresaron en un $38,7 \%$ que la familia y la pareja han influenciado en el uso de los métodos anticonceptivos, especialmente en el grado décimo (58,6\%); sin embargo, los adolescentes encuestados de undécimo manifestaron no sentir vergüenza con la pareja al exigir el condón $(79,4 \%)$.
Se puede señalar, que los adolescentes encuestados manifestaron que por cada acto sexual cambian de preservativo (76 \%) y solo un 11,8\% lo cambia por cada dos actos sexuales. Con respecto, al uso del método de planificación, se observa que la mayoría de los estudiantes lo utilizan cuando van a tener la relación sexual $(37,4 \%)$, mientras que el $15,4 \%$ se prepara con 1 o 2 días antes de la relación sexual y el $47,3 \%$ lo utilizan teniendo en cuenta el método de uso. Por último, se pudo comprobar que el 56,3\% de los estudiantes manifestaron que usarían métodos anticonceptivos con el fin de prevenir un embarazo, como producto de una vida sexual activa.

\section{Asociación entre características sociodemográficas, conocimientos y prácticas frente a la anticoncepción.}

En las tablas 3 y 4 se presentan los resultados, resaltándose aquellos que fueron estadísticamente significativos, es decir, cuando $\mathrm{P}$ valor fue menor a 0,05. En primer lugar, se puede observar que los conocimientos sobre métodos de planificación familiar se asociaron con aspectos sociodemográficos del estudiante y las prácticas que llevan a cabo. Específicamente las relaciones significativas se dieron entre las siguientes variables:

- Quienes hablaron con la madre sobre planificación familiar mostraron una asociación significativa con el condón como primer método de planificación familiar.

- Con relación al sexo mujer $(\mathrm{p}$ valor $=0,034)$ y la asociación se da con una fortaleza de $14.3 \%$, y con el hecho de haber iniciado las relaciones sexuales $(p=0,049)$, con una fortaleza del $13.5 \%$.

- El conocimiento de los anticonceptivos se relacionó con ambos sexos $(\mathrm{p}=0,001)$ en un 21,2 $\%$.

- De otro lado, el conocimiento sobre métodos anticonceptivos que previenen el embarazo e ITS guardó una asociación significativa con la edad del estudiante 16-18 años ( $\mathrm{p}$ valor $=0,026$ ), con una fortaleza de 19,7\%. Así mismo, este conocimiento tuvo relación con la práctica de cambiar el preservativo por cada acto sexual $(\mathrm{p}=$ $0,005)$, con una fortaleza de $51,6 \%$.

- El sexo femenino con una edad comprendida
E-ISSN 2322-7028

Vol. 15 No. 2

Jul - Dic 2018

Cúcuta, Colombia 
ISSN-PRINT

$1794-9831$

E-ISSN 2322-7028

Vol. 15 No. 2

Jul - Dic 2018

Cúcuta, Colombia entre 16 y 18 años (p valor $=0,004$, fortaleza de la relación $=24,7 \%$ ), manifestó haber practicado relaciones sexuales ( $\mathrm{p}$ valor $=0,002$, fortaleza de la relación $=27 \%$ ), utilizando y/o exigiendo el condón sin vergüenza ( $\mathrm{p}$ valor $=0,038$, fortaleza de la relación $=29,9 \%$ ).

- Tanto el hombre como la mujer reconocieron que ésta podía quedar embarazada una vez realizada la primera relación sexual ( $\mathrm{p}$ valor $=0,024)$ con una fortaleza de $15,6 \%$.

- Por último, tanto las mujeres como los hombres reconocieron que los embarazos pueden ser riesgosos $(\mathrm{p}$ valor $=0,002$, fortaleza de la relación $(21,6 \%)$ y aceptaron que el uso del condón es beneficioso.

- Es de hacer notar, que tanto la mujer como el hombre realizan sus relaciones sexuales ( $\mathrm{p}$ valor $=0,001$, fortaleza de la relación $=23,7 \%$ ), a una edad que fluctúa entre 16 a 18 años ( $\mathrm{p}$ valor $=0,001$, fortaleza de la relación $=26 \%)$. (ver tabla 4$)$

- En la tabla 4 se puede observar que tanto la mujer como el hombre siempre usan el condón como anticonceptivo favorito $(\mathrm{p}$ valor $=0,000$, fortaleza de la relación $=54,4 \%$ )

Tabla 3. Asociación entre conocimientos y prácticas de los estudiantes respecto a métodos de planificación familiar

\begin{tabular}{|c|c|c|c|c|c|c|c|c|c|}
\hline CONOCIMII & NTO & $\mathrm{A}$ & B & $\mathrm{C}$ & $\mathrm{D}$ & $\mathrm{E}$ & $\mathrm{F}$ & G & $\mathrm{H}$ \\
\hline \multirow{4}{*}{$\begin{array}{l}\text { Habla con la madre de } \\
\text { planificación familiar }\end{array}$} & Chi2 & 3,205 & 4,494 & 1,337 & 3,730 & 3,770 & 1,635 & 1,080 & 3,150 \\
\hline & $\mathrm{p}$ valor & 0,201 & 0,034 & 0,513 & 0,049 & 0,438 & 0,897 & 0,299 & 0,369 \\
\hline & V de Cramer & 0,121 & 0,143 & 0,078 & 0,135 & 0,202 & 0,135 & 0,108 & 0,190 \\
\hline & $\mathrm{p}$ valor & 0,201 & 0,034 & 0,513 & 0,049 & 0,438 & 0,897 & 0,299 & 0,369 \\
\hline \multirow{4}{*}{$\begin{array}{c}\text { Conoce sobre } \\
\text { anticonceptivos }\end{array}$} & Chi2 & 2,542 & 10,156 & 2,270 & 1,314 & 1,039 & 0,702 & 2,503 & 0,461 \\
\hline & $\mathrm{p}$ valor & 0,281 & 0,001 & 0,321 & 0,252 & 0,904 & 0,983 & 0,114 & 0,927 \\
\hline & V de Cramer & 0,107 & 0,212 & 0,100 & 0,079 & 0,106 & 0,088 & 0,164 & 0,073 \\
\hline & $\mathrm{p}$ valor & 0,281 & 0,001 & 0,321 & 0,252 & 0,904 & 0,983 & 0,114 & 0,927 \\
\hline \multirow{4}{*}{$\begin{array}{l}\text { Conocimiento del } \\
\text { método anticonceptivo } \\
\text { que previene el } \\
\text { embarazo e ITS }\end{array}$} & Chi2 & 17,422 & 4,379 & 13,728 & 1,490 & 15,266 & 2,582 & 2,034 & 23,714 \\
\hline & $\mathrm{p}$ valor & 0,026 & 0,357 & 0,089 & 0,828 & 0,227 & 1,000 & 0,565 & 0,005 \\
\hline & V de Cramer & 0,197 & 0,140 & 0,246 & 0,084 & 0,403 & 0,168 & 0,146 & 0,516 \\
\hline & $\mathrm{p}$ valor & 0,026 & 0,357 & 0,089 & 0,828 & 0,227 & 1,000 & 0,565 & 0,005 \\
\hline \multirow{4}{*}{$\begin{array}{l}\text { Conocimiento de la } \\
\text { edad desde la que se } \\
\text { pueden usar métodos } \\
\text { anticonceptivos }\end{array}$} & Chi2 & 7,349 & 13,100 & 6,493 & 15,326 & 10,000 & 8,073 & 8,421 & 4,928 \\
\hline & $\mathrm{p}$ valor & 0,290 & 0,004 & 0,370 & 0,002 & 0,616 & 0,921 & 0,038 & 0,841 \\
\hline & V de Cramer & 0,186 & 0,247 & 0,174 & 0,270 & 0,328 & 0,298 & 0,299 & 0,237 \\
\hline & $\mathrm{p}$ valor & 0,290 & 0,004 & 0,370 & 0,002 & 0,616 & 0,921 & 0,038 & 0,841 \\
\hline \multirow{4}{*}{$\begin{array}{c}\text { Conocimiento de si en } \\
\text { la primera relación } \\
\text { sexual puede quedar } \\
\text { embarazada }\end{array}$} & Chi2 & 2,812 & 5,083 & 0,791 & 0,346 & 4,012 & 4,205 & 1,411 & 0,533 \\
\hline & $\mathrm{p}$ valor & 0,245 & 0,024 & 0,673 & 0,557 & 0,404 & 0,520 & 0,235 & 0,912 \\
\hline & V de Cramer & 0,117 & 0,156 & 0,062 & 0,041 & 0,209 & 0,216 & 0,123 & 0,078 \\
\hline & $\mathrm{p}$ valor & 0,245 & 0,024 & 0,673 & 0,557 & 0,404 & 0,520 & 0,235 & 0,912 \\
\hline \multirow{4}{*}{$\begin{array}{c}\text { Reconocimiento de las } \\
\text { edades de embarazos } \\
\text { riesgosos }\end{array}$} & Chi2 & 0,300 & 9,684 & 1,584 & 1,277 & 16,515 & 2,130 & 2,130 & 3,885 \\
\hline & $\mathrm{p}$ valor & 0,861 & 0,002 & 0,453 & 0,258 & 0,001 & 0,831 & 0,144 & 0,274 \\
\hline & V de Cramer & 0,038 & 0,216 & 0,087 & 0,079 & 0,431 & 0,156 & 0,154 & 0,213 \\
\hline & $\mathrm{p}$ valor & 0,861 & 0,002 & 0,453 & 0,258 & 0,001 & 0,831 & 0,144 & 0,274 \\
\hline
\end{tabular}

A: Edad B: Género C: Grado D: Ha iniciado relaciones sexuales E: Condón fue el primer método usado F: Condón es el método anticonceptivo que usa G: Utiliza o exige condón en el acto sexual sin vergüenza

$\mathrm{H}$ : Cambio de preservativo por cada acto sexual

Nota: Las asociaciones significativas con $\mathrm{p}$ valor $<0,05$ están sombreadas

Fuente: Instrumento sobre conocimientos y prácticas relacionados con los métodos de planificación familiar en una institución educativa 
Tabla 4. Asociación práctica y aspectos sociodemográficos de los estudiantes respecto a métodos de planificación familiar

\begin{tabular}{|c|c|c|c|c|}
\hline PRACTICAS & & $\mathbf{A}$ & B & C \\
\hline \multirow{4}{*}{$\begin{array}{l}\text { Ha iniciado sus relaciones } \\
\text { sexuales }\end{array}$} & Chi2 & 14,225 & 11,872 & 3,904 \\
\hline & $\mathrm{p}$ valor & 0,001 & 0,001 & 0,142 \\
\hline & V de Cramer & 0,260 & 0,237 & 0,136 \\
\hline & $\mathrm{p}$ valor & 0,001 & 0,001 & 0,142 \\
\hline \multirow{4}{*}{$\begin{array}{c}\text { Condón fue el primer método } \\
\text { usado }\end{array}$} & Chi2 & 4,722 & 5,031 & 9,403 \\
\hline & $\mathrm{p}$ valor & 0,787 & 0,284 & 0,309 \\
\hline & V de Cramer & 0,225 & 0,233 & 0,316 \\
\hline & $\mathrm{p}$ valor & 0,787 & 0,284 & 0,309 \\
\hline \multirow{4}{*}{$\begin{array}{l}\text { Condón es el método } \\
\text { anticonceptivo que usa }\end{array}$} & Chi2 & 12,618 & 26,969 & 12,251 \\
\hline & $\mathrm{p}$ valor & 0,246 & 0,000 & 0,269 \\
\hline & V de Cramer & 0,372 & 0,544 & 0,365 \\
\hline & $\mathrm{p}$ valor & 0,246 & 0,000 & 0,269 \\
\hline \multirow{4}{*}{$\begin{array}{l}\text { Utiliza o exige condón en el acto } \\
\text { sexual sin vergüenza }\end{array}$} & Chi2 & 1,153 & 0,287 & 1,670 \\
\hline & $\mathrm{p}$ valor & 0,562 & 0,592 & 0,434 \\
\hline & V de Cramer & 0,111 & 0,055 & 0,133 \\
\hline & $\mathrm{p}$ valor & 0,562 & 0,592 & 0,434 \\
\hline \multirow{3}{*}{$\begin{array}{c}\text { Cambio de preservativo por cada } \\
\text { acto sexual }\end{array}$} & Chi2 & 9,070 & 3,987 & 2,596 \\
\hline & $\mathrm{p}$ valor & 0,170 & 0,263 & 0,858 \\
\hline & V de Cramer & 0,321 & 0,213 & 0,171 \\
\hline
\end{tabular}

E-ISSN 2322-7028

Vol. 15 No. 2

Jul - Dic 2018

Cúcuta, Colombia

\section{A: Edad B: Género C: Grado}

Nota: Las asociaciones significativas con $\mathrm{p}$ valor $<0,05$ están sombreadas

Fuente: Instrumento sobre conocimientos y prácticas respecto a la planificación familiar en una institución educativa

\section{Discusión}

La información presentada en páginas anteriores contribuyó a entender la forma en que usan los métodos anticonceptivos los estudiantes encuestados; con estos datos se espera que sirvan de información hacia nuevas investigaciones que contribuyan a la reevaluación y el re-diseño de estrategias de intervención sobre esta temática en ámbitos educativos $(16,17)$.

Cabe destacar, que a partir de este estudio se dedujo que los estudiantes de los grados noveno, décimo y undécimo, oscilaban entre los 13 y 21 años de edad, que procedían de zonas urbanas, son solteros y conviven con sus padres y hermanos, datos que contrastan con los de Jerónimo et al. (18), según el cual los estudiantes pertenecían a instituciones educativas estatales, siendo el 50,5\% de sexo femenino, cursaban cuarto $(53,0 \%)$ y quinto $(47,0 \%)$ año de secundaria y conviven con sus padres y hermanos el $67,9 \%$.

En otro orden de ideas, de la investigación se concluyó que del grupo familiar, la persona con la que en mayor medida hablan los adolescentes sobre planificación familiar es con la madre $(61,8 \%)$, datos que no se relacionan con otros estudios, en donde se reconoce que la comunicación entre padres, madres e hijos, a la hora de tratar temas de salud sexual reproductiva, es deficiente; hecho que se debe fundamentalmente porque muchos de ellos no disponen de información suficiente y adecuada que les permita orientar a sus hijos sobre sexualidad, lo que hace que los jóvenes busquen información a través de amigos o de los medios de comunicación (19). Resultados que son similares a los obtenidos por Jerónimo et al. (18), quienes exponen que la fuente de información primaria de los adolescentes es la madre (34,6\%), seguido de los profesores (28,6\%); también hablan de sexo con el padre $(47,6 \%)$ y con los amigos $(42,7 \%)$.

Sin embargo, Fernández et al. (20), demuestran que el $25 \%$ de los adolescentes indicaron que se sienten "muy incómodos" al hablar con su padre/madre sobre cómo debe comportarse sexualmente. Concluyen que a los/ as adolescentes se les dificulta hablar específicamente 
ISSN-PRINT

1794-9831

E-ISSN 2322-7028

Vol. 15 No. 2

Jul - Dic 2018

Cúcuta, Colombia sobre su conducta sexual, en particular con su padre/ madre (20). Cruces et al. (21) expresan en su estudio que aquellos que han mantenido relaciones sexuales con penetración manifiestan que sus padres tienen un menor nivel de estudios que los que no han mantenido relaciones sexuales $(4.72, \mathrm{p}=0.00)$. También, los que han mantenido relaciones sexuales valoran peor la relación con sus padres y con la familia en general, $(4.77, \mathrm{p}=0.00)$ y $(3.43, p=0.00)$, respectivamente. De igual forma estos alumnos, poca importancia a los valores que les inculcan los padres, al igual que la disciplina/control y comunicación ejercida por ellos (21).

Cortés et al. (22), por su parte aseguran que el comportamiento en cuanto al uso del preservativo o condón para evitar las ITS y embarazos no planificados fue bajo (34\%), en comparación con un alto porcentaje que no estuvo de acuerdo con el uso del condón cuando tienen sus parejas estables.

Por su parte, Palacios et al. (23) estiman que el riesgo sexual, el uso inadecuado del condón y la promiscuidad se asocian significativamente con una mala comunicación con ambos padres; mientras que, Corona y Ortega (24), demuestran que no hubo significancia estadística según los siguientes valores ( $>>0,01$ y $\left.\chi^{2}=3,15 \mathrm{I}\right)$, donde: $46,2 \%$ del total recibió instrucción al respecto $(25,3$ y $20,9 \%$, para chicos y chicas, respectivamente); $35,3 \%$, solo en ciertas ocasiones (15,9 y 19,4\%, en el mismo orden) y 18,4 $\%$ no recibió información alguna $(11,4$ y $6,9 \%$, para varones y hembras); de este modo, se evidencia que es imprescindible la comunicación familiar en el contexto de la salud sexual y reproductiva (25).

De otro lado, los datos obtenidos comprobaron que los adolescentes reconocen tener conocimientos sobre métodos anticonceptivos y los modos de usarlos, debido a que han recibido información al respecto, sobre todo por parte de la institución educativa, calificándola como buena o excelente. Estas cifras fueron notablemente altas, en especial cuando se comparan con otros estudios, como el de Torres (26), quien indicó que sus hallazgos reportaron que el 56,5\% del alumnado nunca recibió información sobre educación sexual, frente al 38,5\% que afirma no tener un conocimiento regular y suficiente sobre enfermedades que se trasmiten por contacto sexual, contrario al 33,5\% que tiene un conocimiento insuficiente; sin embargo, el 54,5\% manifiesta estar bien informado acerca del contagio del VIH, y el
$43,5 \%$ no conocen los métodos de planificación familiar (26).

Por otra parte, Corona y Ortega (24), refieren que la calidad de la información recibida es buena en 54,7 \%, regular en 31,8 \% y mala en $13,4 \%$. Sin mostrar una diferencia estadística significativa ( $\mathrm{p}>0,01$ y $\left.\chi^{2}=1,36\right)$.

Así mismo, Guerrero et al. (27), señalaron que existe un nivel de desconocimiento del $30 \%$ en relación a los métodos anticonceptivos, criterio favorable al aborto en un $88 \%$ de los casos, por tanto existe una baja concienciación respecto a la gravedad e importancia de las ETS. En este mismo estudio se dedujo que las relaciones de pareja son valoradas como absorbentes en un $25 \%$ de los casos, frente a la visión del sexo opuesto que es considerada por estos adolescentes como regular o negativa en casi un 50 $\%$ de los casos. Igualmente, Hernández et al. (28), reseñan que el nivel de conocimiento frente a los métodos anticonceptivos es regular y/o escaso en un $68,3 \%$ por parte del género masculino, con una edad que oscila entre 16 y 17 años, influenciado por ítems que tienen que ver con el compañero sexual, inicio de actividad sexual y método anticonceptivo utilizado.

Se debe resaltar, que los amigos o pares se convierten en la fuente de información principal y ejercen presión para el inicio de relaciones sexuales y uso de métodos anticonceptivos, pues entre amigos y pares existe un alto grado de confianza. En este sentido, Rengifo et al. (29), expresan que el 44,00\% de los adolescentes tienen conocimiento sobre el hecho de que sus pares ya habían iniciado relaciones sexuales. Sin embargo, hay que precisar que los estudiantes de la Institución Educativa en algunos casos no solicitan información sobre métodos anticonceptivos porque esto les genera pena o vergüenza. De otra parte, Binstock y Gogna (30), manifiestan que la dimensión social del adolescente influye en su debut sexual;es decir, que la salida con amigos y el control parental, son elementos que inciden en sus experiencias sexuales, como resultado del contexto relacionado con la crianza y la experiencia diaria del adolescente; lo que significa, que cuanto más joven la adolescente comienza a salir, más tempranamente se inicia sexualmente; aunque se debe precisar que la supervisión familiar condiciona la iniciación sexual (30).

No obstante, estudios como el de Morales et al. (31), han encontrado que existe un alto grado de confianza 
en los padres, porque no creen que sea vergonzoso preguntar (61\%); mientras que, en otros, sí surge el miedo a preguntar por vergüenza o pena; igualmente, creen que sus progenitores no les contestan, que se pueden reír o enojar lo que indica que no existe confianza, y que por tanto es malo, de ahí que lo mantengan en secreto (39\%). Contrario a ello, Bárcena et al. (32), estiman que los padres tienen una gran influencia en las variables mediadoras que influyen en la autoeficacia del adolescente frente al uso de métodos de planificación y sus estereotipos hacia el cuidado de la salud y/o práctica sexual; así pues, los padres mantienen una expectativa positiva hacia la comunicación que establecen con sus hijos sobre relaciones sexuales, sin mostrar miedo o vergüenza (32). En este orden de ideas, Sevilla y Orcasita (33), manifiestan que padres y madres con pocas claves para la interpretación y ponderación de las realidades de sus hijos/as y del peso de sus historias en todo lo relacionado con la salud sexual, ofrecen una información atomizada a sus hijos adolescentes, generando en ellos pánico sexual y discursos contradictorios en la relación padre e hijo (34).

Del mismo modo, se logró demostrar que algunos aspectos en torno al conocimiento sobre métodos de planificación familiar por parte de los estudiantes, se relacionan con prácticas que llevan a cabo. Esto se interpreta en el sentido por el cual el conocimiento guía el actuar de la población, particularmente en relación con los métodos anticonceptivos que previenen el ITS; así como el cambiar de preservativo $(\mathrm{p}<0,05)$, o el hecho de conocer la edad desde la cual se pueden usar preservativos e iniciar la actividad sexual $(\mathrm{p}<0,05)(35)$.

Teniendo en cuenta los datos anteriores, es de hacer notar, que Sánchez y Robles (36), reportan que el inicio de la vida sexual en promedio de un adolescente se da a los 13 años $(D E=1.47)$ y el $45 \%$ planean tener relaciones sexuales, aunque en la práctica sólo un $50.5 \%$ usa condón masculino. Además, demuestran que solo el 43,5\% de los adolescentes reportó haber usado el condón en su última relación sexual (36).

En un sentido similar, el conocimiento y las prácticas suelen asociarse a factores sociodemográficos, como la edad y el género del estudiante, lo que hace concluir que tanto hombres como mujeres tienen concepciones y preocupaciones distintas en torno a la salud sexual y reproductiva y a sus prácticas sexuales; de ahí, que la edad condicionaría el hecho de desarrollar una práctica más segura, basada en la adquisición y apropiación de conocimientos que mejoran con la edad y los procesos afines a la madurez y a la llegada de la adultez $(37,38)$. Así mismo, Sánchez y Robles (36), afirman que al analizar los resultados sobre el nivel de conocimiento $(60.4 \%)$ no se obtuvo significancia con el sexo, el estado civil o la ocupación, ni los grupos por edad $(\mathrm{p}=0.03)$, puesto que los de mayor edad obtuvieron mejores resultados.

Frente a esta problemática el equipo de salud se enfrenta al reto de asumir la educación sexual de la población adolescente, con base en las políticas en salud integral y a los hallazgos del colectivo institucional respecto a la práctica sexual, teniendo en cuenta el riesgo de la misma frente al insuficiente conocimiento de la anticoncepción y la práctica inadecuada en el uso de anticonceptivos.

Para finalizar, es importante destacar que la educación para la salud es la herramienta fundamental de conocimiento, información y apoyo familiar para alcanzar las ventajas de una práctica sexual responsable y evitar problemas sociales de embarazos no planeados o el padecimiento de enfermedades por contacto sexual. Es desde esta perspectiva, que los programas de planificación familiar deben formularse teniendo en cuenta los aspectos culturales de la comunidad, entre ellos, la aceptación masculina de los métodos y la influencia de amigos en la práctica sexual (18).

\section{Conclusiones}

- Partiendo de los elementos previamente mencionados, se concluye que los estudiantes tenían edades promedio entre $13-15$ años y 16 - 18 años, de sexo masculino y femenino, de estado civil soltero, procedente de zonas urbanas, convive con la madre, y en otros casos con padres y hermanos. Además, la madre representa la figura más cercana para conversar temas de planificación familiar.

- Referente a la planificación familiar, los adolescentes tienen conocimientos adecuados sobre la descripción, uso, ventajas y desventajas de los métodos anticonceptivos y su prevención en enfermedades de trasmisión sexual. Frente a
E-ISSN 2322-7028

Vol. 15 No. 2

Jul - Dic 2018

Cúcuta, Colombia 
las prácticas de uso, se percibe que la vergüenza se constituye en una barrera que impide solicitar información sobre los métodos anticonceptivos, no obstante, consideran importante la planificación familiar.

- Atravésdelaasociaciónestadísticaselogródeterminar que los conocimientos, las prácticas y diversas características se relacionaron entre sí, especialmente lo relacionado con la edad, el sexo femenino, el conocimiento de métodos anticonceptivos que previenen el embarazo e ITS, el riesgo de embarazo y el hecho de iniciar de forma temprana la práctica sexual. De igual forma, se subrayó que el preservativo se considera como método de elección confiable en las relaciones sexuales, asociado positivamente entre ser hombre o mujer.

- Asimismo, se destaca que el conocimiento de los métodos de planificación familiar, repercute en el comportamiento de la salud sexual y reproductiva de los estudiantes, sin olvidar la existencia de brechas informativas relacionadas con las redes informativas, la educación para la salud y el uso adecuado de los métodos en la población adolescente.

\section{Agradecimiento}

Agradecemos a las estudiantes: Eliana María De La Hoz Herrera, Yuranis Paola Monterroza Barreto, Wendy Milena Valdés Miranda y Daniela Ester Vega Berdugo.

\section{Conflicto de intereses}

Las autoras declaran no tener conflicto de intereses.

\section{Referencias Bibliográficas}

1. Moya Plata D, Oviedo L, Ariza Ortiz A, Uribe A. Reflexiones sobre conocimientos-prácticas de sexualidad en los estudiantes de la facultad de salud y medicina en la universidad de Santander. Bucaramanga-Colombia. Rev Cuid. [Internet]. 2011 [consultado en enero 15 de 2017]; 2(1):149-154. Disponible en https://www.revistacuidarte.org/index.php/cuidarte/article/view/50/689

2. Chandramohan Sriram. Knowledge among rural women about family planning methods in poonamalle block of thiruvallur district, Tamilnadu: a crosssectional study. Tamilnadu: Indian Streams Research Journal. 2014; 4(1): 2230-7850

3. Ministerio de Salud y Protección Social. Profamilia. Resumen Ejecutivo. Encuesta Nacional de Demografía y Salud [Internet]. 2015 [consultado marzo 23 de 2017]; Disponible en: http://profamilia. org.co/docs/Libro\%20RESUMEN\%20EJECUTIVO.pdf

4. Rengifo Reina HA, Córdoba Espinal A, Serrano Rodríguez M. Conocimientos y prácticas en salud sexual y reproductiva de adolescentes escolares en un municipio colombiano. Rev. Salud Pública. 2012; 14(4):558-569.

5. Vélez Arango AL. El embarazo en el adolescente: una visión desde la dimensión emocional y la salud pública. Rev. Cuid. [Internet]. 2012 [consultado el 24 de marzo de 2017]; 3(3):394-403. Disponible en: http://www.scielo.org.co/pdf/cuid/v3n1/v3n1a20.pdf

6. Lapeira Panneflex P, Acosta Salazar D, Vásquez Munive M. Conocimientos, creencias y prácticas de los adolescentes de la cultura Caribe en anticoncepción. Rev Cuid. [Internet]. 2016 [consultado el 24 de marzo de 2017]; 7(1):1204-1209. Disponible en: https://www.revistacuidarte.org/index.php/ cuidarte/article/view/243

7. República de Colombia. Ministerio de Educación Nacional. Resolución No 3353, 2 de Julio, por el cual se establece el desarrollo de programas y proyectos institucionales de Educación Sexual en la Educación básica del País. [Internet] Santa Fe de Bogotá D.C: Minsalud; 1993. [Consultado el 3 febrero de 2016] Disponible en: http://www.mineducacion.gov.co/1621/articles-172061 archivo pdf_politicaSSR.pdf

8. Estrada F, Hernández Girón C, Walker D, Campero L, Hernández Prado B, et al. Use of family 
planning services and its relationship with women's decision-makingand support from their partner. México: Salud Pública [Internet]. 2008 [consultado 10 de marzo de 2017]; 50(6):472-481. Disponible en: https://www.ncbi.nlm.nih.gov/pubmed/19039436

9. Alemán Rivera IS, Cortés Escárcega I, Pérez Cuevas R. Knowledge. Behaviors and beliefs of family planning methods and sexually transmitted diseases among nursing students. Bol Med Hosp Infant Mex [Internet]. 2013 [consultado el 10 de marzo de 2017]; 70(1):18-23. Disponible en: http://www. medigraphic.com/pdfs/bmhim/hi-2013/hii131e.pdf

10. Monteiro de Castro M, Castro Marcos V, Samartine Júnior H, Carvalho do Lago V. Salud reproductiva en la adolescencia: comunicación, funcionalidad de la familia y comportamiento preventivo. Adolescencia \& Salud. [Internet]. 2014 [consultado el 12 de Abril de 2017]; 11(3):87-95. Disponible en: http://www.adolescenciaesaude.com/detalhe artigo.asp?id=451\&idioma=Espanhol

11. Mendoza LA, Arias GM, Pedroza PM, Micolta CP, Ramírez RA, Cáceres GC, et al. Actividad sexual en adolescencia temprana: problema de salud pública en una ciudad colombiana. Rev. chil. obstet. ginecol. [Internet]. 2012 [consultado 15 de marzo de 2017]; 77(4):271-279. Disponible en: http:// www.scielo.cl/scielo.php?script $=$ sci arttext\&pid $=$ S0717-75262012000400006

12. Hernández Ávila M, Garrido Latorre F, López Moreno S. Diseño de estudios epidemiológicos. Revista Cubana de Higiene y Epidemiologia [Internet]. 2007 [consultado el 15 de marzo de 2017]; 45(1):144154. Disponible en: http://www.redalyc.org/articulo.oa? $\mathrm{id}=223219928010$.

13. Monje Álvarez CA. Metodología de la investigación cuantitativa y cualitativa. [Guía didáctica]. Colombia-Neiva: Universidad Surcolombiana; 2011 [consultado el 7 de noviembre de 2014]. Disponible en: https://www.uv.mx/rmipe/files/2017/02/Guia-didactica-metodologia-de-la-investigacion.pdf

14. Rodríguez Rodríguez M, Rodríguez Sánchez PM, López Sánchez Y, Cúbela Rodríguez M. Pacientes VIH-SIDA: inmunidad y calidad de vida. Manzanillo. 2007-2008. Multimed. [Internet]. 2009 [consultado el 16 de marzo de 2017]; 13(2):41-52. Disponible en: http:/www.multimedgrm.sld.cu/ articulos/2009/v13-2/8.html

15. República de Colombia. Ministerio de Salud. Resolución No. 8430, octubre 4, por la cual se establecen las normas científicas, técnicas y administrativas para la investigación en salud. [Internet]. Bogotá D.C.: Minsalud; 1993 [consultado el 10 de noviembre de 2015]. Disponible en: https://www.minsalud. gov.co/Normatividad_Nuevo/RESOLUCION\%208430\%20DE\%201993.pdf

16. Cuéllar Alméciga A, Garzón Fierro L, Medina Díaz L. Uso de métodos anticonceptivos en adolescentes de una institución distrital de Bogotá, segundo semestre 2008. [Tésis en Internet] Bogotá D.C: Pontificia Universidad Javeriana; 2008.

17. Rodríguez Cabrera A, Sanabria Ramos G, Contreras Palú M, Perdomo Cáceres B. Estrategia educativa sobre promoción en salud sexual y reproductiva para adolescentes y jóvenes universitarios. Revista Cubana de Salud Pública. [Internet]. 2013 [consultado el 9 de marzo de 2017]; 39(1):161-174. Disponible en: http://www.scielosp.org/pdf/rcsp/v39n1/spu15113.pdf

18. Jerónimo Maturana CA, Álvarez León JG, Carbonel Luyo WF. Sexualidad y métodos anticonceptivos en estudiantes de educación secundaria. Acta Med Per. [Internet]. 2009 [consultado el 5 de octubre de 2017]; 26(3):175-179. Disponible en: http://www.scielo.org.pe/pdf/amp/v26n3/a06v26n3

19. Ayres JRCM, Carvalho YM, Nasser MA, Saltão RM, Mendes VM. Caminhos da integralidade: adolescentes e jovens na Atenção Primária à Saúde. Interface [Internet]. 2012 [consultado el 5 de octubre de 2017]; 16(40):67-82. Disponíble em: http://dx.doi.org/10.1590/S1414-32832012005000021

20. Fernández AM, McFarlane MN, Ricardo LD, Betancourt E, Cintrón Bou F, Varas-Díaz N, Villarruel A. Actitudes hacia la comunicación sexual entre padres/madres y adolescentes en Puerto Rico. Rev Puertorriquena Psicol. [Internet]. 2017 [consultado el 30 de abril de 2018]; 28(1):80-95. Disponible en: https://www.ncbi.nlm.nih.gov/pmc/articles/PMC5520656/

21. Cruces Montes SJ, Guil Bozal R, Sánchez Sevilla S, Zayas García A. Contexto comunicativo, familiar 
ISSN-PRINT

$1794-9831$

E-ISSN 2322-7028

Vol. 15 No. 2

Jul - Dic 2018

Cúcuta, Colombia y escolar del adolescente y conducta sexual. Revista INFAD de Psicología. [Internet]. 2017 [consultado el 30 de abril de 2018]; 2(1):31-46. Disponible en: http://dx.doi.org/10.17060/ijodaep.2017.n1.v2.916

22. Cortés Alfaro Alba, García Roche René, Ochoa Soto Rosaida. Sexual behavior and condom use among students from the School of Health Technology. Rev Cubana Med Trop [Internet]. 2015 [Consultado el 30 de Abril de 2018]; 67(2):202-212. Disponible en: http://scielo.sld.cu/pdf/mtr/v67n2/mtr07215.pdf

23. Palacios Delgado J, Bravo Flórez M, Andrade Palos P. Consumo de alcohol y conducta sexual de riesgo en adolescente. Psychology International. 2007; 18(4):1-13.

24. Corona Lisboa J, Ortega Alcalá J. Comportamiento sexual y conocimiento sobre infecciones de transmisión sexual en estudiantes venezolanos de un liceo del municipio de Miranda. MEDISAN [Internet]. 2013 [consultado el 5 de octubre de 2017]; 17(1):78-85. Disponible en: http://scielo.sld.cu/ scielo.php?script $=$ sci arttext\&pid $=$ S1029-30192013000100011

25. Becerra AC, Peña WA. Sexo y sexualidad, complejidad de la identidad humana. Revista Colombiana de Enfermería. [Internet]. 2013 [consultado el 24 de junio de 2017]; 8(8):187-199. Disponible en: http://m.uelbosque.edu.co/sites/default/files/publicaciones/revistas/revista colombiana enfermeria/ volumen $8 / 018$ articulo16.pdf

26. Torres LP. Conocimientos sobre métodos anticonceptivos e infecciones de transmisión sexual en estudiantes del nivel secundario de la localidad de Villa Cañas. [Tesis en Internet] Rosario: Universidad Abierta Interamericana; 2011.

27. Guerrero Masiá M, García Jiménez E, Moreno López A, Conocimiento de los adolescentes sobre salud sexual en tres institutos de educación secundaria valencianos. Rev Pediatr Aten Primaria. [Internet]. 2008 [consultado el 5 de octubre de 2017]; 10(39):433-442. Disponible en: http://www.pap.es/ files/1116-849-pdf/962.pdf

28. Hernández Céspedes JD, Velásquez López RN, Pinzón Gutiérrez CM. Conocimiento, actitud y práctica en anticoncepción en adolescentes escolarizados en la comuna 1 de Villavicencio. Rev. Ciencia y salud virtual. [Internet]. 2017 [consultado en octubre de 2017]; 9(1):4-12. Disponible en: http://revistas. curnvirtual.edu.co/index.php/cienciaysalud/article/view/775

29. Rengifo Venegas S, Uribe Godoy V, Porra Quijandría K. Inicio temprano de relaciones sexuales en adolescentes escolares de la ciudad de Ica, 2014. Rev méd panacea. [Internet]. 2014 [Consultado el 30 de Abril de 2018]; 4(1):8-12. Disponible en: http://revpanacea.unica.edu.pe/index.php/RMP/article/ download/131/106/

30. Binstock G, Gogna M. (2015). La iniciación sexual entre mujeres de sectores vulnerables en cuatro provincias argentinas. Sexualidad, Salud y Sociedad [Internet]. 2015 [consultado el 30 abril 2018]; (20):113-140. Disponible en: https://dx.doi.org/10.1590/1984-6487.sess.2015.20.09.a

31. Morales Díaz E, Solanelles Rojas AM, Mora González SR, Miranda Gómez O. Embarazo no deseado en alumnas universitarias. Rev Cub Med Mil [Internet]. 2013 [consultado el 5 de octubre de 2017]; 42(2):153-163. Disponible en: http://scielo.sld.cu/scielo.php?script=sci arttext\&pid=S013865572013000200004\&lng=es.

32. Bárcena Gaona S, Robles Montijo S, Díaz Loving R. El papel de los padres en la salud sexual de sus hijos. Acta de investigación Psicológica. [Internet]. 2013 [consultado el 4 de octubre de 2017]; 3(1):956-968. Disponible en: https://www.sciencedirect.com/science/article/pii/S2007471913709451

33. Sevilla TM, Orcasita LT. "Hablando de sexualidad": una mirada de los padres y las madres a los procesos de formación con sus hijos/as adolescentes en estratos populares de Cali. av. enferm [Internet] 2014. [Consultado el 30 de Abril de 2018]; 32(2):191-205. Disponible en: http://www.scielo.org.co/ pdf/aven/v32n2/v32n2a03.pdf

34. Allen Leigh B, Villalobos Hernández A, Hernández Serrato MI, Suárez L, de la Vara E, de Castro F et al. Inicio de vida sexual, uso de anticonceptivos y planificación familiar en mujeres adolescentes y adultas en México. Salud pública Méx [Internet]. 2013 [consultado el 5 de octubre de 2017]; 
55(2):S235-S240. Disponible en: http://www.scielo.org.mx/scielo.php?script=sci arttext\&pid=S003636342013000800021\&lng=es.

35. González JC. Conocimientos, Actitudes y Prácticas sobre la Sexualidad en una Población Adolescente Escolar. Rev. Salud Pública. [Internet]. 2009 [consultado el 5 de octubre de 2017]; 11(1):14-26. Disponible en: http://www.scielosp.org/pdf/rsap/v11n1/v11n1a03.pdf

36. Sánchez Medina R, Robles Montijo S. Apoyo, Supervisión y Comunicación con Padres y su Relación con el Comportamiento Sexual de Jóvenes en Conflicto con la Ley. Acta de investigación Psicológica. [Internet]. 2014 [Consultado el 5 de octubre de 2017]; 4(1):1398-1411. Disponible en: http://www. scielo.org.mx/scielo.php?script $=$ sci arttext\&pid $=$ S2007-48322014000100007

37. González Gómez Y. El papel del docente en la educación para la sexualidad: algunas reflexiones en el proceso educativo escolar. Rev. Actualidades Investigativas en Educación [Internet]. 2015 [Consultado el 5 de octubre de 2017]; 15(3):1-15. Disponible en: http://www.redalyc.org/articulo. oa? id $=44741347020$

38. González Zúñiga S, Quezada Ugalde AM. La persona adolescente y su experiencia durante la etapa intermedia. Rev. Enfermería Actual de Costa Rica [Internet]. 2015 [consultado el 18 de septiembre de 2017]; 29:1-14. Disponible en: http://www.scielo.sa.cr/scielo.php?script=sci_arttext\&pid=S1409$45682015000200001 \& \operatorname{lng}=$ en. 